peritoneum or cellular tissue would be under the same conditions. I cite here a case of this class:

J. C., male, aged 27, admitted to Alexian Brothers' Hospital June, 1895 ; three tours previously was shot from in front with a 44-caliber bullet which passed into the chest and fractured the fifth rib; it lodged under the skin behind near the angle of the eighth rib, which was fractured. The first day he was suffering from slight dyspnea, some pain on the right side, had expectorated a small quantity of blood and had pneumothorax. The following day his temperature was 100 degrees, pulse 94 , respiration 32 , tongue slightly coated. At noon of the third day his temperature was 103 degrees, dulness extended up to the axillary line in the recumbent position, tongue slightly coated, and he was mildly delirious; bronchial breathing could be heard over the upper portion of the chest behind; this was probably transmitted. Fourth day-When I arrived at the hospital at 12 o'clock temperature was 105 degrees (rectal). pulse 160 , respiration 42 ; he was cyanotic, unconscious and in a low, muttering delirium. He was placed on the table and without anesthetic a resection of the eighth rib was made at the line of fracture. The bullet and fragment of cloth were removed from the muscle wall, also a large quantity of offensive bloody debris and fluid from the pleural cavity. The open ing at the point of entrance of the bullet was enlarged and an irrigation of the cavity with sterile water was made. The day following his pulse was 132 , temperature 102 degrees, respira tion $36 ;$ his delirium had almost subsided and his general appearance was improved. In another twenty-four hours his temperature had dropped to 100.2 degrees, his respiration 28 and pulse 124, and his general condition was still better. From this time on his improvement was rapid. The drainage tube was removed on the sixteenth day and in three weeks he left the hospital.

This case is typical of its class, and similar cases may be observed almost any time in the hospitals of large cities. The rule is, however, that they are allowed to go on to death without surgical interference, as the following case illustrates:

M. C., age 19, admitted to Alexian Brothers' Hospital May 16, 1893. A 44-caliber bullet penetrated the anterior wall of the right half of the chest fracturing the third rib. The pa. tient was very much shocked from the injury; pulse was 130 ; skin cold and clammy and countenance blanched; anxious expression; there was a marked area of dulness over the lower half of the right chest; pneumothorax of the upper half; hemoptysis was continuous but not severe. The following day the patient's temperature was 101 degrees, pulse 118 , respirations 54. He requested to be discharged from the hospital and under strong pressure was persuaded to remain another day. The third day his respirations were 40 , pulse 150 , temperature 103 degrees. He was delirious. Operation was advised but the parents would not consent. He was taken to his home, where after surgical consultation it was decided that he could live but a few hours and that interference would be futile. The death took place five days after removal from the hospital. The attending doctor told me that his condition remained about the same until the end. The postmortem revealed a penetration of the middle lobe of the right lung and the upper portion of the lower lobe. There was a fracture of the seventh rib at its angle, the bullet had dropped back in to the pleural cavity. The chest was full of an offensive sanguino-purulent fluid there was a cloudy swelling of all the tissues, no metastatic abscesses. The cause of death was acu te septicemia.

This case shows how long a patient, with this variety of infection, may live ufter his condition seems hopeless. I might cite many similar cases from the emergency wards of the county hospital. We hope the surgery of the future will lend a belping hand to stay the mortality from secondary infection in gunshot wounds of the chest. Simple incision and drainage may be all that will be necessary, but there should not be the slightest hesitancy in resecting one or more ribs and making a free incision in the pleural cavity in its most dependent portion regardless of the point of entrance or exit of the bullet. The clinical course and treatment of penetrations with other sharppointed instruments is based on the same pathologic and therapeutic principles as stab and bullet wounds; the lung is rarely injured with these instruments and primary drainage is more frequently indicated.

(To be continued.)

\section{REST-A NEGLECTED FACTOR IN THE TREATMENT OF GASTRO-INTESTINAL DISORDER.}

Presented to the Section on Practice of Medicine at the Forty-ninth Annual Meeting of the American Medical Association. held at Denver, Colo., June 7-10, 189s. BY C. D. SPIVAK, M.D.

LECTURER ON DISEASES OF THE GASTRO-INTESTINAL TRACT, UNIVERSITY OF DENYER, MEDICAL DEPARTMENT.

DENVER, COLO.

It is not within the province of my paper to enter into the discussion of the subject of rest from a physiologic standpoint. The question of rest is only being at present approached by the physiologists of the day through the study of muscle and nerve irritability, waste and repair, sleep and the still lees explored domain of "fatigue." Not until sufficient experimental data will have been accumulated will we be in a position to form a rational physiologic theory of rest. I will use the word "rest" in its most comprehensive sense-the antithesis of action. Life is action, but action is made possible by the period of rest during which the spent energies are renovated. All our organs manifest periods of action and repose alternately; some are absolutely rhythmic, as the circulation of blood; others are only intermittent, as the secretions and excretions. The organs of vegetative life are so wonderfully arranged that they take their rest without asking our permission for it. Nobody can bid the heart beat 120 times a minute, neither is one able to retain his urine at will for one week. It is different with the organs of animal life. Here automatism ceases. If he will he thinks, if he will he exercises his muscles. He knows no limit except the point of fatigue, which is the threshold of the pathologic. There is another difference between the organs of animal and vegetative life. After a contin. uous use of any one of the organs of animal life, be it thought, sensation or contractility of muscles, there comes a period of repose during which the organs of vegetative life go on performing their functions undisturbed. On the contrary, when the organs of vegetative life have overstepped the point of fatigue, the organs of animal life invariably suffer in consequence more or less. It is well known how digestive, circulatory and urinary troubles influence the motor, sensory and intellectual functions. Rest, therefore, can be defined as nature's prophylactic. It not only prevents the further breaking down of tissues, but it affords the chance for the tissues to store up new energy. In the ordinary life of a healthy individual the period of activity and prophylactic rest are balanced unnoticed and unobserved. We are unable to measure the time of activity and rest. But as soon as man deviates from the normal it then becomes evident that the two must remain in a direct ratio in order to establish the equilibrium. The greater the activity that produced certain abnormal phenomena the longer must be the rest. The length of time which is required by nature for the repair of an injury must be in proportion to the severity of the injury, and the more severe the injury the longer time is required for the perfect recovery of the disturbed functions. Rest in such cases becomes a therapeutic agent. 
Prehistoric man, I am sure, did not know of the/different from any other organ in our bodies; they terms prophylaxis, metabolism, katabolism, energy, are made up of the same tissues, are subject to the etc., and yet rest has probably been his chief, and for same insults, and their mode of repair is identical a long time his only, remedy. Nature has insisted with that in any other part of the body. I do not see upon it, "even to the extent of inflicting a twinge of the difference between the symptom-complex called pain on such as disobeyed her precepts." And not. neurasthenia and that symptom-complex called nervous withstanding the fact that the beneficent action of dyspepsia. Why apply the Weir.Mitchell treatment rest is so obvious, and may be said to be inherent and in the one and not in the other? I do not underinstinctive, so much so that even animals employ it as stand by what manner of reasoning one arrives at the a therapeutic agent in surgical as well as medical conclusion that in the treatment of a painful limb, cases, yet it was only in the seventies of this century according to the maxim of Hilton, "the maximum that the first word was spoken in the elucidation of the question of rest. I refer to the classic lectures of John Hilton on "Rest and Pain." The surgeon was the first to recognize the value of rest. In 1878 the neurologist followed and sang the praises of nature's greatest Materia Medica. It was our own WeirMitchell who elaborated in his work, "Rest in the Treatment of Nerve Disorders," a cure that bears his name, the brilliant results of which made its author known wherever medicine is practiced. Since that time it seems as if the surgeon and neurologist have monopolized "rest." It is indeed a happy coincidence that those who suffer from pneumonia and typhoid fever are unable to walk, otherwise the physician would never think of ordering them to go to bed. In our day of multiplicity of drugs, patent and proprietary, legitimate and illegitimate, nostrum and specific, when a patient presents himself, and the diagnosis is once made, the first question is: "Which of the thousand and one drugs shall I prescribe?" Surely there ought to be one which will hit the right point. The surgeon thinks first of all of rest. The neurologist tries to secure rest. The physician alone uses the pharmacopeia first, last and all the time.

True, in looking over the scanty literature on the subject of rest one finds here and there a timid voice raised apologetically for the use of rest in cardiac, pulmonary and renal troubles. But the fear of being branded a crank makes them speak in tones not louder than a whisper, and their effort is lost in the pandemonium of the "new" and "newer remedies" craze.

I wish to raise my voice many octaves above a whisper and proclaim loudly and boldly that I employ rest, nature's greatest panacea, as my first and best weapon against the greatest foe of mankind-diseases of the gastro-intestinal tract. I know that the tendency of our time is to treat diseases of the stomach by washing, scouring, scratching, punching and electrifying the stomach. The gastro-enterologists of the country have at the present time visions bright of the oncoming time when the same modus operandi will be applied to that poor piece of gut which measures only twelve fingers in length-when we will be able to wash, scour, scratch, punch and electrify the duodenum. Far be it from me to scorn the work of an Einhorn, Hemmeter, Stewart or Turck. There is none who appreciates more highly the services they have rendered to gastrology than I do. I can assure you that before my stomach will perform the last functions of autodigestion I will yet wash, scour, scratch, punch and electrify a good many stomachs. But I refer more to the tendency of the time, which makes everyone who reads a book on diseases of the stomach arrive at the only conclusion that lavage is the only salvation of the stomach, and that the stomach tube will "do it all." of result is co-equal with minimum of disturbances," and that a painful stomach or painful intestine should not be accorded the same privileges. And lastly, it is incomprehensible to fathom the wisdom of prescribing rest for the weary limb, an exhausted organ, anywhere in the body except in the stomach. The only redeeming instance in the whole array of diseases to which the stomach is heir, and in which the rest cure is recognized as the remedy par excellence is ulcer of the stomach. The principle of the cure was laid down by Niemeyer, recommended by Ziemssen and especially elaborated by Leube. It is expressed by Ewald in the following words: "I know but one form of treatment which holds out prospects of success . . . and this is the rest cure . . . by which the stomach is protected from all irritating factors as a broken bone is immobilized in plaster."

Now, the surgeon does not draw a line of demarkation between an ulcerated, broken, bruised or simply inflamed limb. Rest is the sine qua non treatment. Go over in your mind the whole gamut of diseases of the stomach from a simple inflammation of the mucous membrane to solution of continuity, and you will find that the process, both pathologic and reparative, is the same. Why, then, not apply among other things the same remedy? I have done so and nature has rewarded me most generously.

How can rest be applied to diseases of the stomach and intestine, will be asked. It is true you can not put these organs in splints, but you can, by using one or all of the following methods, secure rest: 1. Rest in bed. 2. Diet. 3. Hot poultices.

1. Rest in bed.--In severe cases I follow the method of Weir-Mitchell to the letter: 1. Absolute rest in bed. Sitting is not allowed under any circum. stances. The bowels are regulated; the bed pan is used. 2. Sponging the whole body every morning. 3. Isolation. 4. Massage. I have not had yet a case that required electricity. In milder cases I use my judgment as to isolation and massage.

2. Diet.--In many severe cases of gastro-intestinal disorders the best bill of fare is abstinence. One, two or even three days' fasting will do no harm in cases of ulcer, dyspepsias, and diarrheas of all kinds and varieties. Nutritive enemata can be employed in cases where a longer period of fasting is necessary. When food by the mouth is allowed it must be given in small quantities, no matter whether liquid or solid, and at regular intervals. Every case must be individualized as to the quality and quantity of food.

3. Poultices.-I shall not attempt to formulate a theory as to the action of poultices. Whether the poultices hasten the expulsion of food from the stomach as has been shown experimentally by Fleischer, or they cause the acceleration of the circulation of the blood in the abdominal viscera, is not yet definitely settled. I am certain, however, of one

I take the stomach and intestine to be in no way thing, and that is that they aid the peristaltic move- 
ments of the stomach, they make the patient feel comfortable and keep him warm and at rest. The poultices take the place of splints. In severe cases the poultices are applied constantly during the day for one and two weeks. In milder cases from four to eight hours daily.

The time allotted to me does not permit me to enter into details. Each of the three forms by which rest of the gastro-intestinal tract may be secured would require a separate essay. I will therefore limit myself to the recital of the histories and results of a few cases which have undergone the rest cure.

Case 1.-Referred to me by Dr. J. T. Eskridge. J. H. S., Plattville, Colo., aged 31 years, male, merchant. Family history negative. Had continued fever in 1887, lasting for two weoks. In $1890 \mathrm{had}$ influenza. These two attacks left him in a weakened condition. For the last years the following train of symptoms have developed gradually : Headache almost constant, at times accompanied with pain in back; distress and heaviness after meals; burning sensation in stomach from one to two hours after ingestion of food, which is sometimes relieved by taking baking soda, of tener not. Appetite is good, bowels regular; has not lost perceptibly in body weight; suffers occasionally from jerking and twitching of muscles, has attacks of despondency and melancholy and spells of crying; once in three to four days, sometimes at longer intervals, becomes dizzy when commencing his meals; sleeps well ; suffers from nocturnal emissions quite frequently; attacks of frequent urination. Physical examination of mouth, chest and abdomen negative, except a slightly coated tongue, and some tenderness over pit of stomach and transverse colon. Repeated examinations of stomach contents after various meals, at various times revealed a total acidity varying from 90 to 140 . Not being able at that time to absent himself from home and to take the rest cure, he was ordered to subsist on nitrogenous diet; he was given strychnin in small doses, sod. bicarb. after each meal, lavage every other day. His symptoms were greatly mitigated. The headaches diminished both in frequency and severity; he could concentrate his mind; his weight increased. Yet he was not cured from the burning sensation in his stom. ach. He had to take soda every day, and at times even large quantities would not relieve.

Six months after he first consulted me he decided to try the rest cure. In addition to absolute rest in bed he had hot poultices applied to his abdomen twice a day, cold sponging every morning and his diet regulated. $\mathrm{He}$ was not given any soda, neither was his stomach washed. The burning sensation gradually diminished and at the end of fourteen days he was entirely free from this annoying symptom. Contrary to my advice that he remain in bed at least two weeks longer, in order to insure a permanent cure, he returned to his home. He informs me (now one year after the treatment) that he is doing well, and that only within the last two months he has once in a while the recurrence of slight attacks of heartburn. Case 2.-J. O., female, 24 years of age. Married four years. Has no children. Parents healthy. Was well until five years ago, when she had an attack of influenza which left her with a bad cough, and she gradually grew worse. She came from Portland, Ore., to Denver two years ago. She markedly improved. For the last three months she commenced to cough and the expectorations were at times blood-streaked, Bloating and pressure after meals very annoying. At first she vomited after meals once or twice a week; later it became more frequent. Can not keep anything on her stomach for the last five days. She has retained, however, her appetite for food. She suffers from night sweats and violent attacks of coughing. Physical examination revealed dullness over the apex of and scattered areas of moist râles heard over the left lung, anteriorly and posteriorly. Thestomach contents showed a great quantity of mucus and diminished acidity. She was sent to St. Joseph's Hospital ; absolute rest enjoined and car. ried out. No food was given for the first twenty-four hours. For the next two days she was allowed small quantities of somatose. Hot poultices applied to abdomen constantly for first four days, then six hours daily. Cod liver oil inunctions daily. No medicines were given by the mouth, except a hypo dermic injection of sirychnin once a day. At the end of four weeks she ate meat at each meal and drank two quarts of milk without experiencing any distress after; has gained six pounds in weight, no night sweats, no harassing cough. She became pregnant five months after she was discharged, miscarried on the third month, and at the time of this writing, fourteen mouths after the treatment, continues in good health.
Case 3....Referred by Dr. S. A. Fisk. J. H. P.; 25 years old ; student. Father's mother, and sister of patient died of lung trouble. Has had lung trouble for two years. Dr. Fisk informed me that he has "considerable infiltration on the right side of his chest, and some beginning on his left; a mitral systolic lesion with a slightly enlarged heart." For four months suffers from diarrhea; has from four to five watery stools daily. No pain in abdomen; appetite fair. Pulse 110, emperature elevated in the afternoon.

Treatment.-Absolute rest in bed. No food for twentyfour bours. Somatose for two days, and after that scraped meat and kephir. For the first five days gave orphol in five grain doses, and washed the bowels twice. After that gave strychnin, one-thirtieth of a grain three times daily. He was in bed three weeks. The diarrhea never returned. Four months after he was discharged Dr. Anderson of Colorado Springs, referred him to me for the examination of his feces, which contained some peculiar extraneous matters. As his feces did not contain at that time anything peculiar, I have examined a specimen under the microscope and found numerous ova of tænia solium. By appropriate treatment have removed without any trouble a tape worm. The patient is now (twenty months after treatment) in Switzerland and has not had an attack of diarrhea since.

Case 4.-Referred by Dr. Clayton Parkhill. F. F. ; 44 years old ; female. Father subject to rheumatism; mother died of a paraly tic stroke. On Dec. 7,1896 , noticed a growth in the left breast, which was diagnosed by Dr. Parkhill as cancer, and which he removed. Six weeks after the operation commenced to feel pain in the epigastric region, aggravated by taking food, which continued for the last five months. Belches a great deal. Appetite fair ; bowels costive. No nausea, no vomiting, no heartburn. Test meal found well digested. $\mathrm{HCl}$ present.

Treatment. - Rest in bed, poultices to abdomen. Meat diet and kephir. Nux vomica fifteen drops three times daily. The pains gradually subsided, and after three weeks she left the hospital cured.

Case 5-Referred by Drs. Eskridge and Wetherill. E. S. ; 42 years old ; female; single. Family history negative. At 9 years of age she had scarlet fever, since which time all her troubles date. She had attacks of dysentery periodically. Intense headache, front and nape. Frequent attacks of diarrhea preceded by pain in intestines, usually over colon. Great irritability in rectum and bladder. In 1891 had a cystic tumor removed from her right breast. In June 1894 had her uterus gcraped, which relieved in a measure the irritability in the bladder and rectum. Four months after the operation a tape worm was removed. In February 1895 hysterectomy was performed and the right ovary removed. The annoying symptoms were not abated in the least. In June 1895 right nephrorrhaphy was performed. In September 1896 she came under my observation, Dr. Wetherell having, after careful examina. tion, refused to operate on her rectum, which he found in a normal condition. After watching her symptoms for some time I have come to the conclusion that she suffers from mem. branous enteritis, and probably has suffered from the same malady all her life. Her treatment consisted of absolute rest in bed, hot poultices to abdomen, and high irrigation in colon with a solution of resorein. Her diet consisted of meat, kephir, bread and plenty of butter. She showed marked improvement from the start, and life was no longer a curse to her. I can not claim that she is entirely cured. The pains in her abdomen recur once in a great while, and she then sheds a good amount of membrane, but the intervals of freedom from pain and annoyance are long, sometimes lasting four and five weeks, and the attacks are never as intense. She has taken three courses of rest cure, each continuing five, four and three weeks respectively.

Case 6.-.-Referred to me by Dr. D. H. Coover, D. H. C. ; 38 years old; married. Father and mother of gouty disposition. Enjoyed fair health when a girl. Having resided in a malarial region, she suffered from intermittent fever. For the last fifteen years suffered intensely from pain in abdomen and diarrhea. She was never free from pain more than a few days at a time; suffered from neuralgic pain in head and sciatic nerve. As far back as 1885 she noticed in her evacuation shreds of mucous membrane, sometimes a full cast of the bowel. During the last few months the pains became unbear. able; she could nut sleep, was despondent and very miserable. She came under my notice June 1897.

Treatment-Rest cure absolute, hot poultices. No food for the first twenty-four hours, and small quantities of somatose during the next four days. High irrigation of the bowel, with resorcin solution at first every day for five days, then once a week ; five grain doses of orphol. At the end of the first week 
she was rewarded for her enforced rest by having a spontaneous, well-formed stool, an event which has not occurred for many years. She remained in bed for three weeks. Four weeks after she had a slight relapse and was again ordered to remain in bed, and this time she remained for about four weeks. At the time of this writing, one year after the rest cure, she is in good health; has not had a single attack of diarrhea or abdominal pain.

Case 7 -Referred by Dr. S. A. Fisk. R. S. ; 39 years old; female; Mexican. Three years ago had a bilious attackheadach $\theta$ diarrhea and vomiting, lasting for one day. Since that time suffers from diarrhea. Has one well formed stool every morning, and four to five evacuations during the day, the last stool usually containing some mucous and shreds of mem. brane. She has never had a movement during the night. Stools are watery and very offensive. She has no pain in abdomen, no pressure or distress. Has lost twenty pounds in wejght since her first attack. Took a course of treatment in Carlsbad and Paris, with only slight and temporary relief. She came under my treatment in September 1897. I have pursued the same course of treatment as in No. 6. Being of a highly nervous temperament, as soon as the diarrhea stopped she left for Tucson, Arizona, where she kept up the rest cure for some time. Dr. Rodgers of Tucson, under whose care she was, writes to me that she has no diarrhea and that her stools are perfectly normal.

Case 8.-Miss M. G, ; 27 years old ; medical student. Parents healthy. She enjoyed good health until within six months, when she commenced to feel pain in her stomach after meals. Pain is localized, aggravated by pressure and by taking food, and even water. The pain grew gradually worse, and her phy sician had recourse to opiates. No nausea, no vomiting, no heartburn, no belching, no headache. Appetite good; bowels regular. HCl 80. She remained in bed only ten days, as she was anxious to come up for examination in May (1898). Hot poultices were applied six hours daily. Somatose given dur ing the first three days, and later small quantities of milk and lime water every half hour. At the end of the ten days she was free from pain. At the time of this writing, four weeks af ter the rest cure, she complains of slight pain once in two or three days.

Case 9.-Referred by Dr. Mullin of Aspen, Colo. M. B. 52 years old; female; German. Family history negative. Fourteen years ago had an attack of typhoid fever. Seven years ago, immediately after drinking a cold glass of beer, was taken sick. At first she felt as if there was a load in her stom ach, which continued for several days. She suffered on and off from abdominal pain, since. In 1893 had severe pains, and her physician washed her stomach and put her on a milk diet, which relieved her very much. Whenever she would have an attack this mode of treatment was employed with success. In January 1898 had influenza, and since that time the condition of stomach became worse. Pain is aggravated by taking food, by exercise, and is worse toward evening. The pains are especially severe from 8 to 11 P.M. No headache. Appetite fair. Bowels have been always regular; constipated since the attack of influenza. She came under my treatment May 14 1898. The stomach contents revealed nothing abnormal. On palpation a tumor was found in the right abdominal cavity, movable on respiration, and making excursions from under the ribs down almost to the brim of the pelvis. The diagnosis of a morable right kidney was substantiated by Dr. C. A. Powers.

Treatment-Absolute rest in bed, hot poultices, massage, cascara sagrada (Stearn's) for the constipation, and strynchin. The pain gradually diminished. After the first week she has not had a single severe attack. Sleeps well, Her appetite is excellent, and if she remains in bed for three weeks longer, I expect,' that with an abdominal bandage so fitted as to keep the kidney in situ, she will remain comfortable through her life.

$$
\text { ANALYSIS OF THE CASES. }
$$

I have chosen from my records of over one hundred cases treated by rest-cure during the last two years these nine cases with a view of illustrating the groups of diseases wherein I found my method to give satisfaction.

Case 1 represents a type of hyperacidity upon a nervous basis. Washing of the stomach and alkalies were of no avail. Being a case of neurasthenia with hyperacidity only as a predominating symptom, rest logically suggested itself to my mind.

1 My prognosis came true. Patient was discharged cured, July 1.
Cases 2 and 3 represent the usual type of tubercular patients, the first suffering from vomiting, the other from diarrhea, both conditions being analogous, and only differing from one another as to the seat of the irritability in the digestive tract. To let such patients eat, drink and walk about is simply to add insult to injury. All around rest for body, stomach and intestine is clearly indicated.

Cases 4 and 9 represent types in which the suspicion of cancer is very strong. Case 4 had a cancer removed from her breast and case 9 had all the symptoms diagnostic of cancer, her age, the constant pain, the emaciation. The first case proved to be a severe dyspepsia, the other a floating kidney.

Cases 5, 6, and 7 represent various types of enteritis. It is my belief that through a mistaken diagnosis case 5 was unsexed and otherwise mutilated.

Case 6 convinced me that all cases of enteritis without an organic lesion are amenable to treatment, and case 7 presents a point of interest that membranous enteritis does not necessarily follow the letter of the text-books, and may be present without severe pain.

Case 8 represents a type of cases in which the diagnosis of ulcer or erosion of the stomach is very probable but not certain. The age of the patient, the localized pain increased by pressure and by the taking of food, and the slight hyperacidity all point to ulceration or erosion. The diagnosis may be doubtful, but the beneficent result of the rest treatment is certain.

\section{CONCLUSIONS}

1. It is indicated in all dyspepsias, the underlying cause of which is a deranged nervous system.

2. It is indicated in all cases where abdominal pain is present.

3 . In all cases of acute and chronic diarrhea.

4. In hemorrhage from the stomach or intestines.

5 . In all cases of moveable or floating kidney.

6 . In all tubercular cases suffering from disturbed digestion, be it stomach or intestine, and especially those that have a vacillating temperature record, and as there are but few cases of tuberculosis that do not suffer from some form of gastro-intestinal disorders, the rest cure is indicated in 50 per cent. of all cases of tuberculosis. Not only are they relieved from their annoying digestive symptoms, but their general condition markedly improves.

7. I have yet to learn of a disease of the gastrointestinal tract where the rest treatment is contradicted.

I will close my paper by a quotation from Hilton's "Rest and Pain," which I have altered somewhat to meet the occasion. "In all diseases of no matter what nature, of the gastro-intestinal tract, when the evidence of disease is in organic lesion or in deranged function, it becomes our duty to look upon and treat the altered structures as we do contusions and laceration of soft parts and congestion of organs in any other part of the body, and to give the stomach and intestines absolute rest, to rely on nature's power to repair the injury or disturbance, and to avoid stimu. lants, which excite rapid circulation as much as possible. The stomach and intestines disturbed in their vital endowment become unequal to even their ordinary duties. They recover themselves slowly; they then soon become fatigued from use, and if claims are made upon them soon after injury, that is, before structure and physiologic integrity are re-required, the patient is very likely to suffer from serious dis- 
ease of the alimentary tract. The stomach, as well as the intestines, requires absence from occupation, rest, and it should be in proportion to the severity and duration of the symptoms they present." BIBLIOGRAPHY.

Articles "Repos" and "Loi d'Intermittence," Dictionnaire de Médicine (Littre's), Paris, 1886; article "Repos" in Dictionnaire des Sciences Gould's lictionaries.

Belknap, L. J.: The rest cure, the cases selected; method of treatment, Mich. Med. Society, Detroit, 1892, xvi, p. 161 .

Battey, R.: Rest, the great panacea for human ills, Trans. Med. Associa tion, Georgia, Atlanta, 1877.

Blatchford, T. W.: Report on rest and the abolition of pain in the treat ment of disease, Tr. Med. Society, N. Y., Albany, 1856.

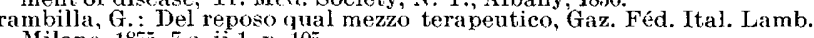
Milano, 18 , r

bilite dans le traitement des affections chirurgicales, paris, 1844

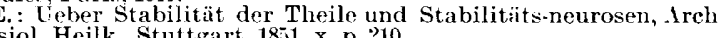
1. physio. Heilk., Stut tgart, 1851, x, p. $\geq 10$.

Journal 1891 rest as a therapeutic agent in

Dippe, E.: Ruhe als Wundheimittell, Schmiedeberg, 1876

Dyson, W.: Some difficulties met with in the employment of rest as a therapeutic agent in medical cases, Med. Press and Circ., London, 1884 , n. s. xxxvii, p. 43 .

Finney, J. M.: Rest, a therapeutic agent in the treatment of diseases more particularly of diseases of the circulatory system, Dublin, Journal Med. Se., 1874, lvii, p. 108.

Hilton, J.: On rest and pain, Lancet, London, 1861, ii ; 1862, $\mathrm{i}$ and ii ; also New York, 187

Hammond, W. A.: How to rest, N. Am. Review, N. Y., 1891, cliii, p. 215. Jackson, A.: Changed aspects of unchanged truths; the use and abuse of rest, Med. Press and Circular, 1885, n. s. xxxix, p. 807

Jacobi, Mary Putnam: Rest, for women.

mes, R.: On the so-called a buse of rest, Liverpool Med. Chirur. Journal, 1886 , vi, p. 18.

sur le movement et le repos, Memoire de l'Acad. Rh. de hirurg., 1779

disease, $1879, i$, p. 47. Maryland Med. Jour., Baltimore, 1891-92, to which

Page, F. W.: Permanency of the rest treatment, Boston Med and Surg. Journal, 1882, cvii, p. $77-81$.

Pope, C.: A plea for the more systematic care and attention of chronic function disease by the rest treatment, Charlotte (N. C.) Med. Journal, 1896 , ix, p. 444-446.

Reyne: Memoir sur le movement et le repos, Mem. de l'icad., Roy. de

Rambaud, $1780 .:$ Du repos envisage comme moyen therapeutic, Paris, 1871. Roberts, F. T. On rest and position in the treatment of medical diseases, Liverpool Med. and Surg. Reporter, 1868, ii, p. 39

Sinkler, W.: Diseases and conditions to which the rest treatment is adapted, Jour . Ner. and Mental Diseases, N. Y., 1892, xix, p. 321-338.

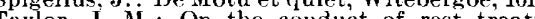

Therapeutic Gaz. DISCUSSION.

Dr. H. M. McConnell of Penngylvania-I do not think there is any class of cases that require rest so much as diseases of the stomach. Rest of this organ means physiologic rest. The only way to obtain this rest is to abstain from food entirely and this can only be done to a very limited extent, when it can be carried out at all. When the Doctor gave the history of his cases he did not say what they were being treated for; in come cases it is injudicious to give nitrogenous food. Some symptoms are due to auto-intoxication, and you must improve digestion in treating these cases. Take ordinary typhoid fever, a patient that takes three or four pints of milk a day is not giving the organs physiologic rest. When you take into consideration imperfect digestion, most cases of neurasthenia are cases of auto-intoxication. Physiologic rest is very important.

Dr. Tyson of Philadelphia-In speaking upon the rest treat. ment of gastro-intestinal troubles the Doctor has spoken of the one thing that will do more or less good in every case of these troubles; the application of therapeutic measures in these gastro-intestinal troubles is more or less haphazard. Those who look into the subject do not receive much assistance so far as therapeutic measures go. I hope the time will come when accurate methods will produce more satisfactory results. In comparison to the rest treatment of the stomach, the Weir Mitchell method is rather analogical. In my experience food is not such a necessary factor in the treatment of these conditions as many would imagine. A patient may go one, two or three days without the ingestion of food, if he is in a position not to demand any force; food is a force-producing agent largely.

Dr. H. A. West of Galveston, Texas-The scope of Dr. Spivak's paper is very comprehensive, because the gastrointestinal canal undergoes serious involvement in nearly all infectious diseases, such as rheumatism, scarlet fever, measles and diseases of that class, in which the processes of digestion are seriously involved. Rest treatment is of supreme value, not only rest so far as can be accomplished by rest in bed, but by other means. And especially as regards acute rheumatism, the prognosis in this disease as regards the subse- quent cardiac complications can be made less or even averted by such a plan of rest in bed. The whole subject is so com prehensive that one can not discuss it in the five minutes allowed him. One point would require modification as to the necessity of rest in all cases of tuberculnsis. If the rest in these cases is limited to the organs of digestion and to the proper regulation of the diet, then I agree with the Doctor but the large proportion of cases is benefited by exercise in the open air.

Dr. SpIvak of Colorado-I have little to say in addition to what I have already said. I am thankful to Dr. Tyson for taking. up the defense. I read the paper not so much to relate to you the nine cases as to urge upon you the necessity of rest in these cases. I wanted to arouse interest in the profession to one of the best methods that can be used and one which nature employs to cure disease. 1 have been inter ested in this subject many months. I am sorry that the facilities of the library here does not permit of more extended research on this subject; there are only fifteen or eighteen papers or monographs in the whole literature on the subject of rest. I have not found one school that treats of the subject of rest in gastro.intestinal disorders. I have seen good results in many cases and in none have I seen any harm result. In regard to tuberculosis, I did not mean to say that all cases should be treated by rest in bed; I said that those cases that were suffering from fever should go to bed. My opinion is based upon a good many cases observed. They feel a great deal better in bed than they do after a walk. Every patient who has a fever, when he exerts takes something away from his strength. Furthermore, I stated that patients who suffered from diarrhea or vomiting should also go to bed. I limit these cases where rest in bed is indicated in tuberculosis where there is temperature, diarrhea and vomiting.

\section{DILATATION OF THE STOMACH, WITH REPORTS OF CASES TREATED BY DIET, MASSAGE AND INTRA. GASTRIC ELECTRICITY.}

Presented to the section on Practice of Medicine at the Forty-ninth Annual Meeting of the American Medical Association, held at Denver, Colo., June 7-10, 1898. BY BOARDMAN REED, M.D. PHILADELPHIA, PA.

There has been a tendency of late among writers to regard enlargements of the stomach as of little or no consequence, unless very excessive and complicated by such a degree of muscular weakness as to render the organ incapable of emptying itself at any time during the twenty-four hours. Some recent authors do not give any place to dilatation of the stomach as a distinct or separate entity, but describe it incidentally under the heads of motor insufficiency and obstruction at or near the the pylorus, on the ground that it is a mere sequela or consequence of one of these. But, notwithstanding the high authority which exists for a contrary view, the writer insists that there is a normal size for stomachs as much as for hearts, and that any notable enlargement of either, plus decided insufficiency, constitutes dilatation. It is granted that it is not in either case a primary disease, and also on account of the enormously disturb. ing influence of unhygienic diet and dress, stomachs do differ very widely in size within so-called normal limits; but even the cases called megalo-gastria by Ewald,' in which in consequence of excessive eating or drinking the stomach is often found twice the usual size without indigestion, are no more normal than are hypertrophied hearts in which the circulation is well maintained. The enlarged stomachs which have not yet dilated are, in fact, very frequently a cause of other pathologic conditions and of symptoms both direct and indirect, especially by displacement of the uterus.

But to follow up this line of discussion would lead too far afield. The object of this paper is to show 